\title{
Vulvar cancer staging: guidelines of the European Society of Urogenital Radiology (ESUR)
}

\author{
Olivera Nikolić ${ }^{\dagger \dagger}$, Filipa Alves e Sousa $2^{2^{*}}$ (D) Teresa Margarida Cunha ${ }^{3}$, Marijana Basta Nikolić ${ }^{1}$, \\ M. Milagros Otero-García ${ }^{4}$, Benedetta Gui ${ }^{5}$, Stephanie Nougaret ${ }^{6}$ and Henrik Leonhardt ${ }^{7}$ On behalf of the ESUR \\ Female Pelvic Imaging Working Group
}

\begin{abstract}
Objective: The aim of the Female Pelvic Imaging Working Group of the European Society of Urogenital Radiology (ESUR) was to develop imaging staging guidelines for vulvar cancer and to propose standardised MRI protocols and reporting.

Methods: The guidelines recommended from the ESUR in this article resulted from a questionnaire analysis regarding imaging staging of vulvar cancer that was answered by all members of the Female Pelvic Imaging Working Group. Only the answers with an agreement equal to or more than $80 \%$ were considered. Additionally, the literature was reviewed to complement and further support our conclusions.

Results: The critical review of the literature and consensus obtained among experts allows for recommendations regarding imaging staging guidelines, patient preparation, MRI protocol, and a structured MRI report.

Conclusions: Standardising image acquisition techniques and MRI interpretation reduces ambiguity and ultimately improves the contribution of radiology to the staging and management of patients with vulvar cancer. Moreover, structured reporting assists with the communication of clinically relevant information to the referring physician.
\end{abstract}

Keywords: Vulvar cancer, Staging, Magnetic resonance imaging, Guidelines, Protocol

\section{Key points}

- MRI is the modality of choice for local staging of vulvar cancer.

- T2WI, DWI-MRI, and DCE-MR are recommended.

- The most widely accepted criterion for inguinofemoral lymphadenopathy is short-axis $>1 \mathrm{~cm}$.

- The most specific criterion for inguinofemoral lymphadenopathy is the presence of necrosis.

*Correspondence: filipasousa039@gmail.com

${ }^{\dagger}$ Olivera Nikolić and Filipa Alves e Sousa share first authorship

2 Department of Radiology, Centro Hospitalar Universitário de Lisboa

Central, Alameda Santo António Dos Capuchos, 1169-050 Lisboa,

Portugal

Full list of author information is available at the end of the article

\section{Introduction}

Vulvar cancer is a rare gynaecologic malignancy, representing only $2-5 \%$ of cases, primarily affecting postmenopausal women [1]. Initial diagnosis is made by gynaecological examination and punch/incision biopsy. Squamous cell carcinomas (SCC) account for the vast majority of vulvar cancers ( $>85 \%)$ [2]. The International Federation of Gynaecology and Obstetrics (FIGO) [3] and the TNM classification [4] systems are both used to stage vulvar cancer and are closely aligned. The final diagnosis is established by histological examination of the primary tumour and lymph node specimens [5].

In vulvar cancer, metastatic involvement of the inguinofemoral lymph nodes is the most important 
prognostic factor and influences the surgical approach and the need for chemoradiation therapy $[1,6]$.

Imaging modalities, such as ultrasound, computed tomography (CT), combined ${ }^{18} \mathrm{~F}$ fluorodeoxyglucose positron emission tomography and CT (FDG-PET/CT), and magnetic resonance imaging (MRI), are not an integrated part of staging according to FIGO, nevertheless they are well-recognised to provide valuable information concerning local tumour status, lymphadenopathy, and distant metastasis. Clinical or imaging suspicion of lymph node involvement should be further analysed by fine-needle aspiration (FNA) or core biopsy whenever this additional information impacts the primary treatment choice [5].

Due to its excellent contrast resolution, MRI is considered the imaging modality of choice for evaluating local growth of vulvar cancer and to exclude invasion of nearby situated organs. In spite of that MRI staging of vulvar cancer is not used routinely in all cancer centres, and it could be argued that MRI is only indicated for larger tumours [7]. Furthermore, despite its wide utilisation, a lack of standardised recommendations/guidelines for MR protocol and reporting is notable.

The aim of this manuscript is to present the ESUR recommendations for the initial staging of vulvar cancer, based on recent clinical and imaging developments. The value of an appropriate MR imaging protocol and standardised imaging reports is highlighted.

These guidelines apply to adults over the age of 18 who have SCC of the vulva and do not address patients with other vulvar cancer histologies.

\section{Material and methods}

\section{Questionnaire and consensus meeting}

A Questionnaire consisting of 54 questions was designed by the authors and then sent out to the Female Pelvic Imaging Working Group for approval. Indications and technical details, including minimal hardware characteristics, patient preparation, examination protocol and reporting were analysed. For some questions multiple answers were possible. Not all the questions were answered by all the participants. A total of 21 responses were obtained and analysed. Each item was classified as follows: (1) "RECOMMENDED" (at least $80 \%$ agreement in favour), (2) "NOT RECOMMENDED" (at least $80 \%$ agreement in opposition) or (3) "UNCERTAIN", i.e. consensus was not reached (less than $80 \%$ agreement). The results were presented to and discussed with the ESUR Female Pelvic Imaging Working Group. The panel included 21 experts from 20 different institution in Europe: Portugal $(n=3)$, France $(n=2)$, Spain $(n=2)$, United Kingdom $(n=3)$, Germany $(n=1)$, Austria $(n=1)$, Sweden $(n=1)$, Italy $(n=2)$, Serbia $(n=2)$, and
Greece $(n=2)$. Two panelists were from two institutions outside Europe: Japan $(n=1)$ and USA $(n=1)$. The panel's recommendations (based on at least $80 \%$ consensus among experts) are given in Table 2.

\section{Literature search}

We searched the PubMed/Medline database, using the following search terms: vulvar cancer; vulvar carcinoma; gynaecologic malignancies; ultrasound; computed tomography; magnetic resonance imaging; and positronemission tomography/computed tomography (PET/CT). We selected relevant English-language papers on vulvar cancer, with a special focus on its imaging evaluation.

\section{Role of imaging in staging vulvar cancer Primary tumour staging}

Due to its excellent contrast resolution and the ability to depict perineal and vulvar anatomy to great detail (illustrated in Fig. 1), MRI is the imaging modality of choice for the local staging of vulvar cancer.

No relevant literature was found regarding MRI in the evaluation of primary tumours $\leq 2 \mathrm{~cm}$, confined to the vulva and/or perineum, and with $\leq 1 \mathrm{~mm}$ of stromal invasion. As such, MRI is not recommended in these cases.

Pelvic MRI including the inguinal regions should be performed for local staging of SCC with stromal invasion $>1 \mathrm{~mm}$, tumour size $>4 \mathrm{~cm}$ or tumours with suspicious involvement of the urethra, vagina, or anus according to clinical evaluation $[8,9]$. A lack of evidence regarding the appropriateness of MRI staging of tumours sized between $>2 \mathrm{~cm}$ and $\leq 4 \mathrm{~cm}$ with stromal invasion $\leq 1 \mathrm{~mm}$ is noted and, in those cases, the decision to refer the patient to MRI should depend on the clinical suspicion of tumour invasion of the nearby situated organs.

In a study including 22 patients prior to surgery, MRI accurately staged primary tumour extent ( $\mathrm{T}$ stage) in $70 \%$ of patients (Sohaib et al. [10]). In another study, tumour size was correctly assessed in $86 \%$ of cases, with both unenhanced and contrast-enhanced MRI, and the overall staging accuracy was $69.4 \%$ for unenhanced MRI increasing to $85 \%$ with the addition of contrast-enhanced sequences (Kataoka et al. [11]).

\section{Lymph node status and distant metastases}

Recurrence in the inguinal lymph nodes carries a very poor prognosis, with most cases resulting in the patient's death within 1 year; therefore, evaluation of nodal status at initial staging and adequate groin treatment is determinant for prognosis and overall survival [12].

First-line evaluation of inguinal lymph node involvement is clinical inspection and palpation, and a positive evaluation should prompt further imaging examination 


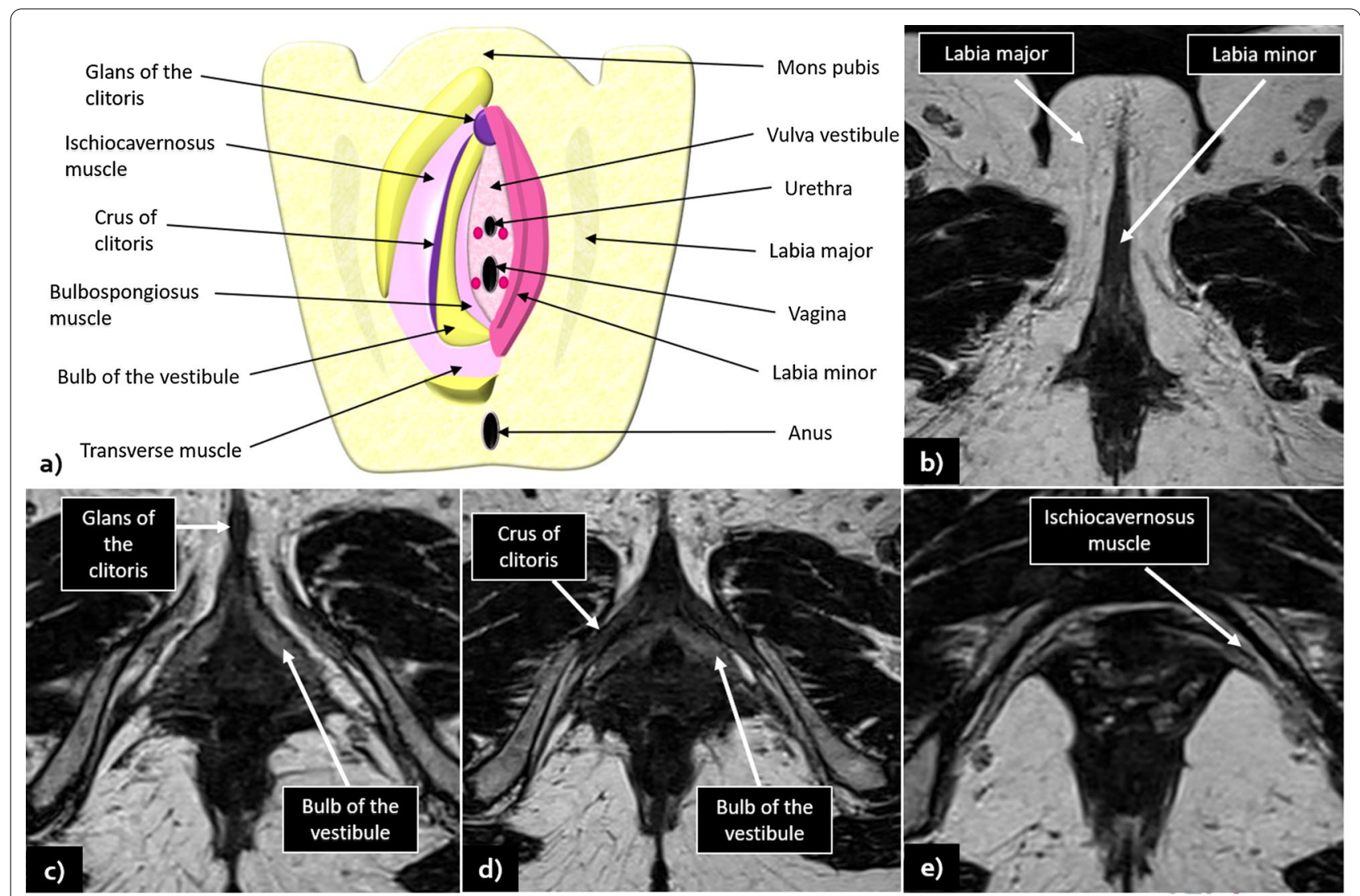

Fig. 1 a Schematic illustration of vulvar anatomy: b-e show MRI normal findings and vulvar anatomy in axial T2WI sequences

irrespectively of the primary tumour size and/or stromal invasion depth [5].

Several studies aimed to evaluate the accuracy of different imaging modalities (including MRI, ultrasound with or without fine-needle aspiration (FNA), CT and PET) in assessing metastatic involvement of inguinofemoral lymph nodes in vulvar cancer. (The results of these studies are summarised in Table 1.)

For all imaging modalities, the most commonly used criterion for regional lymph node metastasis is the short-axis, usually considered suspicious when $>1 \mathrm{~cm}$; however, its reported sensitivity is low, ranging between 43 and $86 \%$ [11, 16, 17, 23, 24]. Other features may be helpful, especially when combined, namely irregular contour, round shape, presence of necrosis, loss of fatty hilum and a ratio of short-to-long-axis diameter $\geq 0.75$. Lymph node necrosis demonstrated the highest specificity among individual criteria; however, it has low sensitivity $[11,12]$. Care should be taken when MRI is performed shortly after a diagnostic vulvar biopsy, as this may result in reactive lymph node changes that may be mistaken by metastatic lymphadenopathy yielding a false-positive result [15] - awareness of this possibility and consultation of the cytological/histological results from the (recently) biopsied lymph node should be sufficient to avoid this misdiagnosis.

Several studies have analysed the added value of CT in the staging of primary vulvar cancer. In four prospective studies [21, 22, 24, 25] that aimed to investigate if preoperative CT influences surgical treatment planning, the authors concluded that preoperative CT scanning is of limited value and has no clinical impact as a routine examination, suggesting that it may be omitted in early stage vulvar cancer. On the other hand, in cases of locally advanced disease or in the presence of pathologically proven tumour spread to the inguinal or iliac lymph nodes, further staging with contrast-enhanced $\mathrm{CT}$ of chest, abdomen, and pelvis may provide valuable information and is recommended [5]. Within the major studies $[16,19]$, the coverage of the CT scans varied, including either the abdominal region or the chest and abdominal regions, but always including the pelvic and inguinal regions. All CT scans were performed with contrast enhancement. 


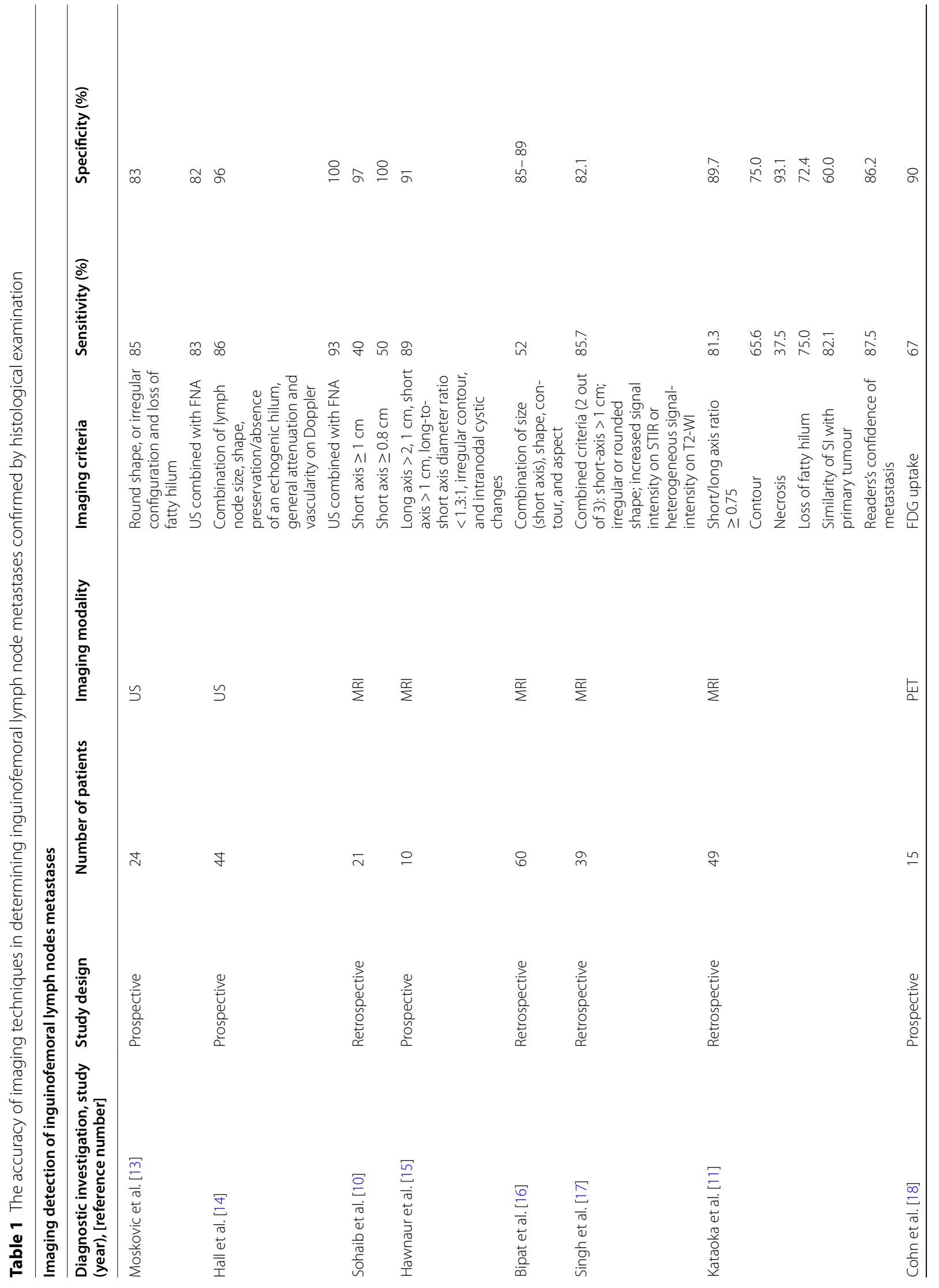




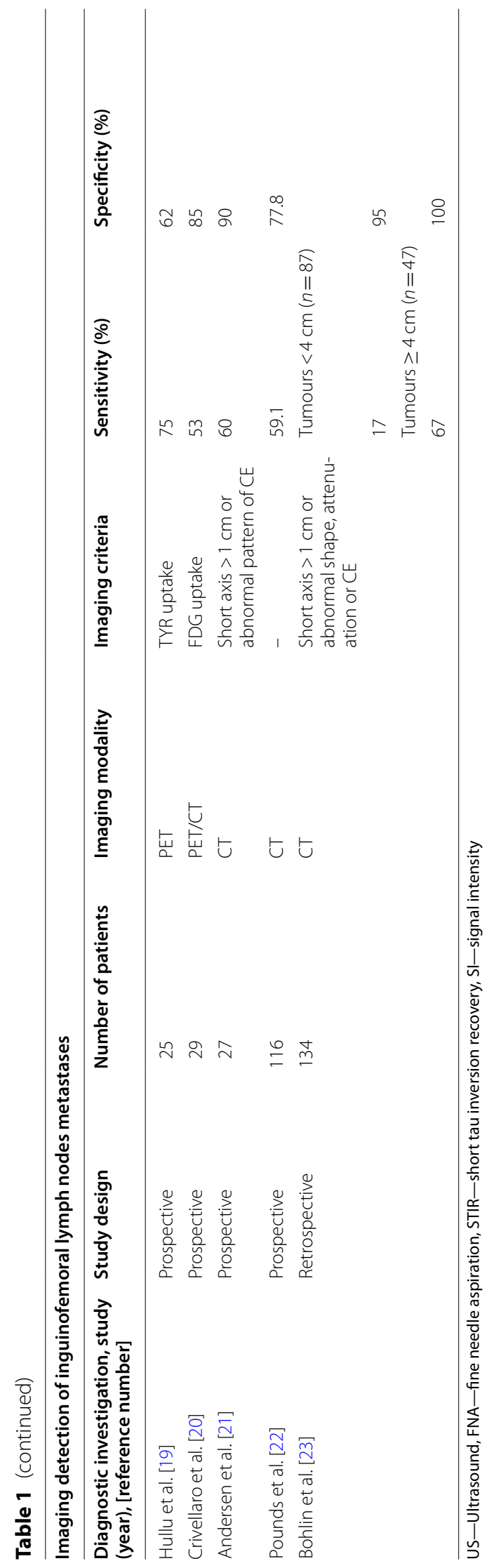




\section{Sentinel lymph node biopsy}

Traditionally, groin treatment in early stage vulvar cancer has included inguinofemoral lymph node dissection (IFLD), which involves the removal of superficial inguinal and deep femoral lymph nodes. While this is an effective approach in promoting survival, it carries a significantly higher risk of complications (such as lymphedema) with increased short- and long-term morbidity when compared to debulking of clinically involved lymph nodes or sentinel lymph node (SLN) [20]. Since only $25-35 \%$ of women with early stage vulvar cancer have groin metastases, IFLD may be considered an overtreatment in most of these cases [21].

In order to avoid unnecessary IFLD, several prospective multicentre trials have evaluated the safety and validity of SLN procedure in early stage vulvar cancer. A multicentre observational study [22] was conducted on 403 women who had primary vulvar tumours with less than $4 \mathrm{~cm}$ in size and depth invasion of more than $1 \mathrm{~mm}-$ inguinofemoral lymphadenectomy was performed only in patients with a positive SLN. With a median follow-up period of 35 months (24-month minimum), groin recurrences were detected in six of the 259 patients (2.3\%) with negative SLN and the 3-year survival rate was $97 \%$. There was a significant reduction in short- and long-term morbidity in cases where only the SLN was removed in comparison with SLN removal followed by IFLD. The long-term follow-up of the GROINSS-V observational study [23], which was also performed on this cohort, compared the results of SLN-positive patients (followed by IFLD) with SLN-negative patients (no IFLN dissection) in a total of 377 patients. At a median follow-up of 105 months, they found no significant differences $(p=0.03)$ in the overall local recurrence at 5 years $(24.6 \%$ for SLN-negative and $33.2 \%$ for SLN-positive patients) and at 10 years (36.4\% for SLN-negative and $46.4 \%$ for SLN-positive patients). Isolated groin recurrence rate was $2.5 \%$ for SLN-negative patients and $8.0 \%$ for SLNpositive patients at 5 years. Disease-specific 10 -year survival was $91 \%$ for SLN-negative patients compared to $65 \%$ for SLN-positive patients $(p<0.0001)$.

A systematic review and meta-analysis [24] of the cumulative data on SLN biopsy in women with unifocal tumours measuring less than $4 \mathrm{~cm}$ and without clinically suspicious inguinofemoral nodes found no significant differences in the rate of groin recurrence after SLN biopsy (3.4\%) in comparison with complete IFLD (1.4\%). In addition, a recent systematic review by a European expert panel [25] concluded that SLN correlates with a low groin recurrence rate and a good 5-year disease-specific survival rate in negative SLN patients, and therefore SLN is currently considered the standard procedure in well-selected women with clinically unsuspicious lymph nodes.

\section{ESUR guidelines}

For primary tumours $\leq 2 \mathrm{~cm}$, confined to the vulva and/ or perineum, and with $\leq 1 \mathrm{~mm}$ of stromal invasion, imaging staging is not recommended. Pelvic MRI including the inguinal regions should be performed for local staging of SCC with stromal invasion $>1 \mathrm{~mm}$, tumour size $>4 \mathrm{~cm}$, or tumours with suspicious involvement of the urethra, vagina, or anus according to clinical evaluation. For tumours $>2 \mathrm{~cm}$ and $\leq 4 \mathrm{~cm}$, clinical staging and groin ultrasound (with puncture of suspicious lymph nodes) or MRI staging are both considered valid options.

For regional or locally advanced disease (FIGO stages III-IVA) or suspicious distant metastases (FIGO stage IVB), chest, abdominal and pelvic CT (or PET/CT) with coverage of the inguinal regions should be performed. Intravenous contrast should be administrated with image acquisition on portal-venous phase (60-80 s) to increase diagnostic accuracy.

The MRI recommendations on imaging of primary vulvar SCC are given in Table 2. Fasting and administration of anti-spasmodic agents are recommended, similarly to the ESUR guidelines for other gynaecologic conditions. The bladder should be emptied before imaging, since a fully distended bladder may inhibit both the degree of straining and the descent of pelvic organs [26]. Vaginal opacification with gel is optional. Future studies may help to establish the added value of vaginal gel in diagnosing small vulvar lesions and early vaginal invasion.

T2WI (T2-weighted imaging), DWI-MR (diffusionweighted imaging magnetic resonance) and DCE-MR (dynamic contrast-enhanced magnetic resonance) are now recommended for the initial staging of vulvar cancers. Contrast-enhanced sequences depict vulvar cancers as early arterial enhancement lesions and can better delineate tumour invasion of the urethra, clitoris, vagina, or anus [12, 27]. Ideally, T2WI and DWI-MR should have the same acquisition plane, field of view, and slice thickness to allow side-by-side interpretation and/or image fusion as this improves diagnostic performance. T2WI with fat suppression may improve the detection of small tumours [2, 12, 27]; however, its usefulness is not consensual among experts, and therefore, it remains optional.

Further, T2WI sequences of the pelvis with a reduced field-of-view (rFOV) are advised since reducing the FOV increases spatial resolution and allows better anatomic detail, which may help in both the detection of small tumours and in delineating tumour invasion of nearby perineal structures [12]. These T2WI sequences with a rFOV may be obtained in axial oblique (perpendicular to 
Table 2 Summary of the recommendations based on $\geq 80 \%$ agreement among experts

ESUR recommendations

\section{Recommendations for MRI staging of vulvar cancer}

\section{- Indications}

Tumour stromal invasion $>1 \mathrm{~mm}$

Tumour size $>4 \mathrm{~cm}$

Tumours with close proximity to or involvement of the urethra, vagina, or anus

\section{- Patient preparation:}

Fasting is recommended $(4-6 \mathrm{~h})$

The use of antiperistaltic agents is recommended (20 mg butyl scopolamine IM/IV or $1 \mathrm{mg}$ of glucagon IV) unless their use is contraindicated due to patient medical background

Supine patient positioning is recommended

Vaginal gel is optional

Rectal gel is not recommended

\section{- Hardware:}

The minimal recommended magnet field strength to stage vulvar cancer is 1.5 Tesla

\section{- Sequences and imaging planes:}

\section{Pelvis}

T1WI

Axial T1W Dixon sequence

T2WI

Axial, sagittal, and coronal two-dimensional T2W sequences

$\mathrm{T} 2 \mathrm{~W}$ sequence with fat suppression is optional

Slice thickness $\leq 4 \mathrm{~mm}$

T2WI with a small FOV (from the vaginal top to the entire perineum included)

Axial or axial oblique (perpendicular to the urethra) and coronal or coronal oblique (parallel to the urethra)

Slice thickness $=3 \mathrm{~mm}$ is recommended

DWI-MRI

In the axial plane, with a minimum of two $b$-values (low $b=0-50$ or $100 \mathrm{~s} / \mathrm{mm}^{2}$, high $b \geq 800 \mathrm{~s} / \mathrm{mm}^{2}$ )

DCE-MRI

Three-dimensional (3D) spoiled gradient-echo fat-suppressed T1-weighted imaging (3DT1WI FS) imaging on axial or axial oblique before and after the administration of intravenous contrast for three scans to obtain arterial, portal and equilibrium phases (the last acquisition may be obtained in the most informative plane for each particular case)

Upper abdomen (to evaluate the Kidneys and lymph nodes)

T2W HASTE axial from the renal hila to the inguinal region

DWI axial from the renal hila to the inguinal region

\section{Recommendations for CT staging of vulvar cancer}

\section{- Indications}

Regional or locally advanced disease (FIGO stages III-IVA) or suspicious distant metastases (FIGO stage IVB) —alternatively to CT, PET/CT may be performed in these cases

\section{- Protocol}

Chest, abdominal and pelvic CT with coverage of the inguinal region after the administration of intravenous contrast with image acquisition on portalvenous phase (60 - 80 s)

\section{Recommendations for inguinofemoral lymph node US and biopsy}

\section{- Indications}

Ultrasound of the inguinal regions with biopsy of suspicious lymph nodes (either by FNA or core biopsy) should be performed in all patients with either clinical (palpation) or radiological suspicion of lymph node metastasis depicted on MRI, CT, or PET/CT

the urethra) and coronal oblique (parallel to the urethra) planes.

For the benefit of spatial resolution with the possibility to reconstruct the acquired images in any desired plane, DCE sequences should be obtained using threedimensional (3D) spoiled gradient-echo fat-suppressed T1-weighted imaging (3D T1WI FS) imaging on axial or axial oblique plane (perpendicular to the long axis 
of the urethra) on pre- and post-contrast administration, for three scans to obtain arterial, portal and equilibrium phases with the last acquisition obtained in the most informative plane for each particular case (usually in the sagittal or coronal plane to add a different perspective from the already acquired sequences with maximum resolution).

Imaging of the upper abdomen to evaluate the kidney and lymphadenopathy is recommended and should include T2W HASTE and DWI in the axial plane from the renal hila to the inguinal region.

\section{MRI structured report}

Unanimous agreement was reached amongst panel members on the need for a structured MRI report in order to improve the report quality and to assist with the communication of clinically relevant information to the referring physician [28-32]. The recommended structured report is given in Table 3 .

\section{Diagnosis and initial staging}

Squamous cell carcinoma (SCC) is by far the most frequent malignant vulvar tumour. According to the latest World Health Organization (WHO) Classification of Tumours [33], SCCs must be classified on the basis of their association with the human papillomavirus (HPV) infection into SCC HPV-associated (having vulvar intraepithelial neoplasia (VIN) as a precursor lesion) or SCC HPV-independent (having differentiated VIN as a precursor lesion, often in association with lichen planus and lichen sclerosus). HPV-independent vulvar SCC has a worse prognosis than HPV-associated vulvar SCC, higher recurrence rates and a greater tendency to rapid progression [33]. In HPV-associated tumours, multifocal lesions and concomitant cervical neoplasia are more frequently observed [33, 34].

In most cases, patients are present at an early stage with vulvar tumefaction or ulcer that may be associated with pain, pruritus, bleeding, or discharge [27, 35].
Asymptomatic cases are less frequent. Diagnosis is histological and should be established with an incision biopsy [5].

MRI, ultrasound with or without puncture of inguinofemoral lymph nodes, CT and PET/CT may be used to define the extent of tumour and/or for treatment planning $[11,20,21,39,40]$.

Lymph node biopsy may be performed either by FNA or by core biopsy. There are no published data comparing the performance of these to puncture techniques in the clinical setting of vulvar cancer staging, and the wider available experience addressing this topic comes from breast cancer studies [36-41]. In the study of Solon et al. [36], core biopsy of suspicious nodes showed a sensitivity rate of $96 \%$, specificity of $100 \%$, positive predictive value of $100 \%$, and negative predictive value of $64 \%$. All these data are superior to previously published studies on ultrasound-guided FNA, which have a sensitivity ranging from 50 to $80 \%$. Moreover, false positive cytology and inadequate sampling are points of weakness of FNA. While the superiority of core biopsy over FNA in vulvar cancer staging has yet to be confirmed by specific prospective trials comparing these diagnostic techniques, in the author's opinion, core biopsy should be preferred whenever possible to obtain sufficient material for histological analysis, although FNA can be considered appropriate for small suspicious lymph nodes.

The most widely used staging system for vulvar cancer is the one developed by the International Federation of Gynaecology and Obstetrics (FIGO) [42], which was revised in 2009 in close collaboration with the American Joint Commission on Cancer (AJCC) and the Union of International Cancer Control (UICC), and is given in Table 4. Major changes of this revision include the combination of the former stages I and II, subclassification of regional lymph node involvement based on the number and size of lymph nodes and the presence/absence of extra-capsular spread, as well as disregard for bilateral lymph node involvement [33]. These changes have been

Table 3 Recommended MRI structured report in vulvar cancer staging

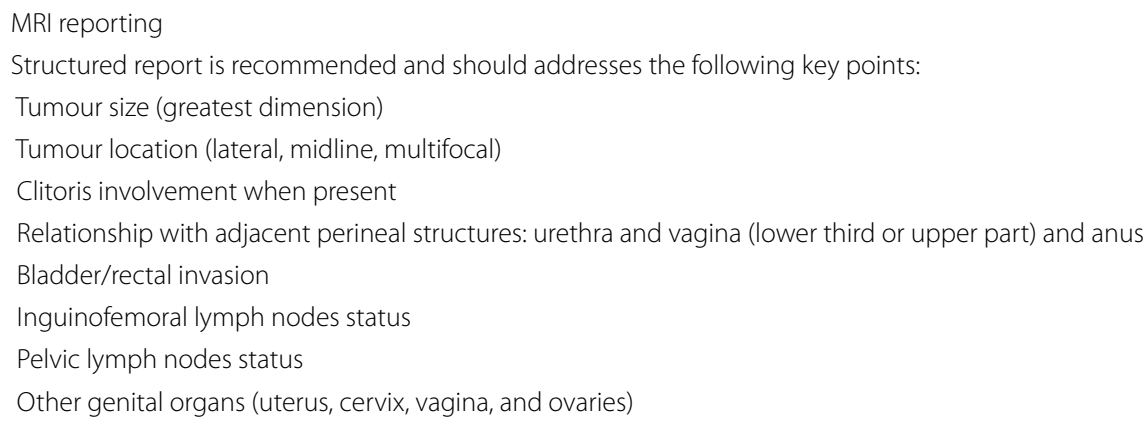


Table 4 FIGO 2009 classification for vulvar cancer staging

\begin{tabular}{ll}
\hline FIGO stage & Description \\
\hline I & Tumour confined to the vulva (without nodal metastasis) \\
IB & Lesions $\leq 2 \mathrm{~cm}$ in size with stromal invasion* $\leq 1 \mathrm{~mm}$ \\
II & Lesions $>2 \mathrm{~cm}$ in size with stromal invasion* $>1 \mathrm{~mm}$ \\
& Tumour of any size with extension to the adjacent perineal structures (lower third of urethra, lower \\
III & third of vagina, anus) without nodal metastasis \\
& Tumour of any size, with or without extension to adjacent perineal structures (lower third of ure- \\
IIIA & thra, lower third of vagina, anus) with positive inguinofemoral nodes \\
& 1. With 1 lymph node metastasis ( $\geq 5 \mathrm{~mm})$, or \\
IIIB & 2. With $1-2$ lymph node metastases $(<5 \mathrm{~mm})$ \\
IIIC & 1. With 2 or more lymph node metastases ( $\geq 5 \mathrm{~mm})$, or \\
IV & 2. With 3 or more lymph node metastases ( $<5 \mathrm{~mm}$ ) \\
IVA & With positive nodes with extracapsular spread \\
IVB & Tumour invades any of the following: \\
\hline
\end{tabular}

"The depth of invasion is defined as the measurement of the tumour from the epithelial-stromal junction of the adjacent most superficial dermal papilla to the deepest point of invasion

validated in several studies [3, 43, 44]. Complete staging using FIGO classification requires primary tumour resection and inguinofemoral lymphadenectomy; however, common practice has evolved to include the use of SLN biopsy as an alternative to complete lymph node dissection, as well as radiological assessment to determine local disease extension, with special emphasis to MRI [34, 45].

Typically, initial treatment of vulvar cancer consists of complete surgical excision, with or without adjuvant radiation therapy (RT) and/or chemotherapy depending on pathology and disease extension [33] (see section "Vulvar cancer: management and treatment" later on this article for a detailed discussion on treatment planning and current guidelines).

\section{FIGO stage I}

Stage I is defined as a tumour confined to the vulva or perineum without lymph node or distant metastasis. It is further sub-divided into stages IA and IB according to tumour size and stromal invasion (Fig. 2):

- Stage IA-Lesions $\leq 2 \mathrm{~cm}$ in size with stromal invasion $\leq 1.0 \mathrm{~mm}$.

- Stage IB-Lesions $>2 \mathrm{~cm}$ in size or with stromal invasion $>1.0 \mathrm{~mm}$.

The role of imaging is limited in stages IA and IB. Vulvar carcinoma is depicted as a solid mass with nonspecific low signal intensity on T1WI and intermediate to high signal intensity on T2WI. DWI-MRI demonstrates restricted diffusion as a high signal intensity lesion on high $b$-value images with low signal intensity on the corresponding apparent diffusion coefficient (ADC) maps. DCE-MR imaging sequences with early arterial phase tumour enhancement may be useful in the detection of small vulvar lesions [12] (Fig. 3). T2WI with fat suppression may also be a helpful sequence, as the perineal region is rich in fat with high-signal intensity on T2WI, and its suppression may make small vulvar lesions more conspicuous $[2,12,27]$.

\section{FIGO stage II}

Stage II is defined as a tumour of any size with extension to adjacent perineal structures (1/3 lower urethra, $1 / 3$ lower vagina, anus) without lymph node or distant metastasis (Fig. 4).

On T2WI sequences, local tumour invasion may be depicted by disruption of the hypointense signal that circumscribes the urethra and/or interruption of the low signal intensity of the vaginal wall or the anal sphincter by an intermediate to high signal intensity tumour [27]. Large tumours may demonstrate high signal intensity on T2WI sequences due to internal necrotic changes [12]. DCE-MRI increases the staging accuracy and can better demonstrate involvement of the urethra, anus, and vagina [11, 27] (Fig. 5).

\section{FIGO stage III}

Stage III represents inguinofemoral nodes involvement irrespective of tumour size or local extension. It is further subdivided according to the number and size of the lymph nodes involved, as well as the presence/absence of 


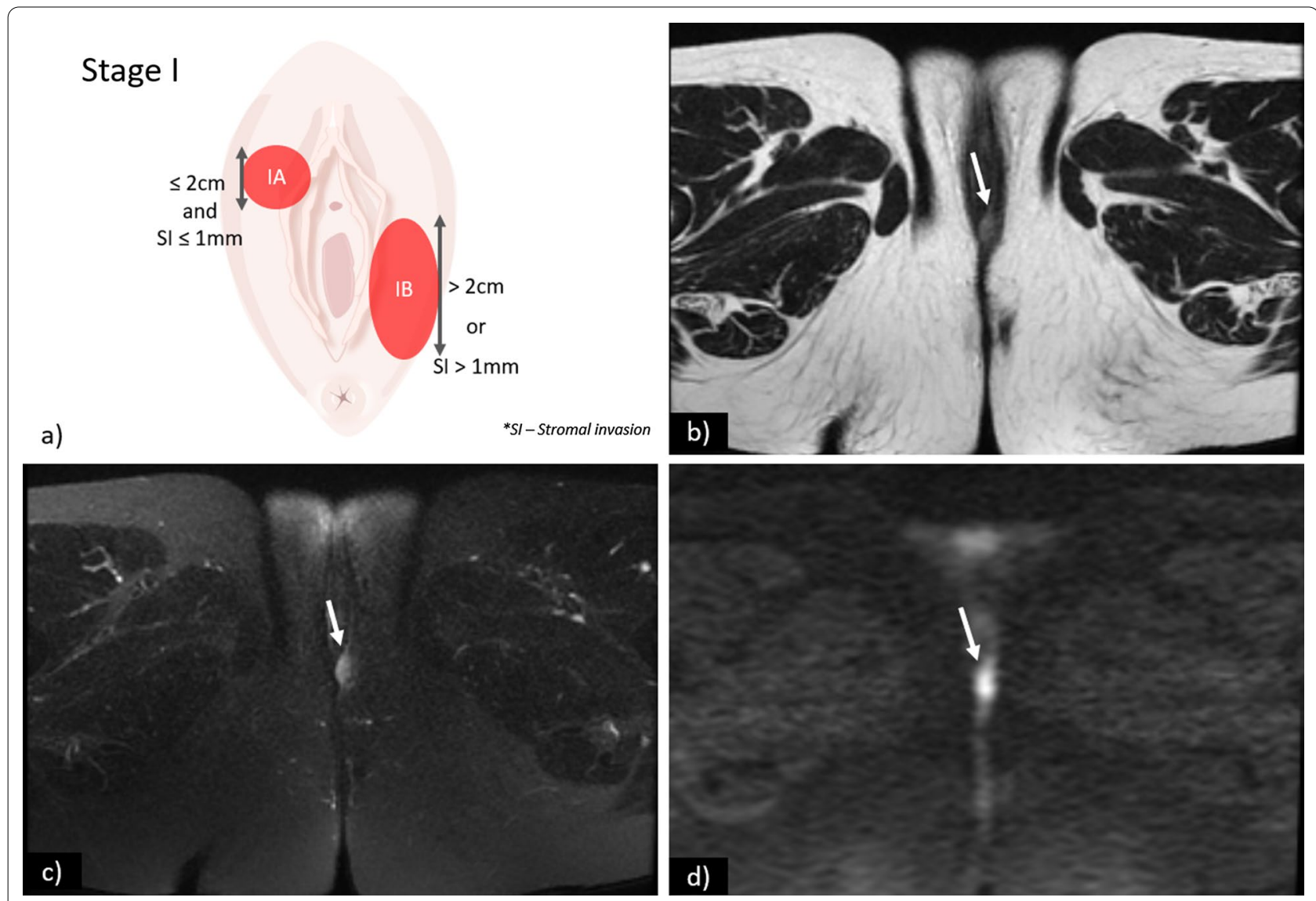

Fig. 2 a Schematic illustration of FIGO stage I. Axial T2WI (b), axial fat saturation T2WI (c) and DWI with $b$-value $=800 \mathrm{~s} / \mathrm{mm}^{2}(\mathbf{d})$ shows a vulvar tumour measuring $<2 \mathrm{~cm}$, with pathologic proven stromal invasion of $4 \mathrm{~mm}$, corresponding to FIGO stage IB. SI_Stromal invasion
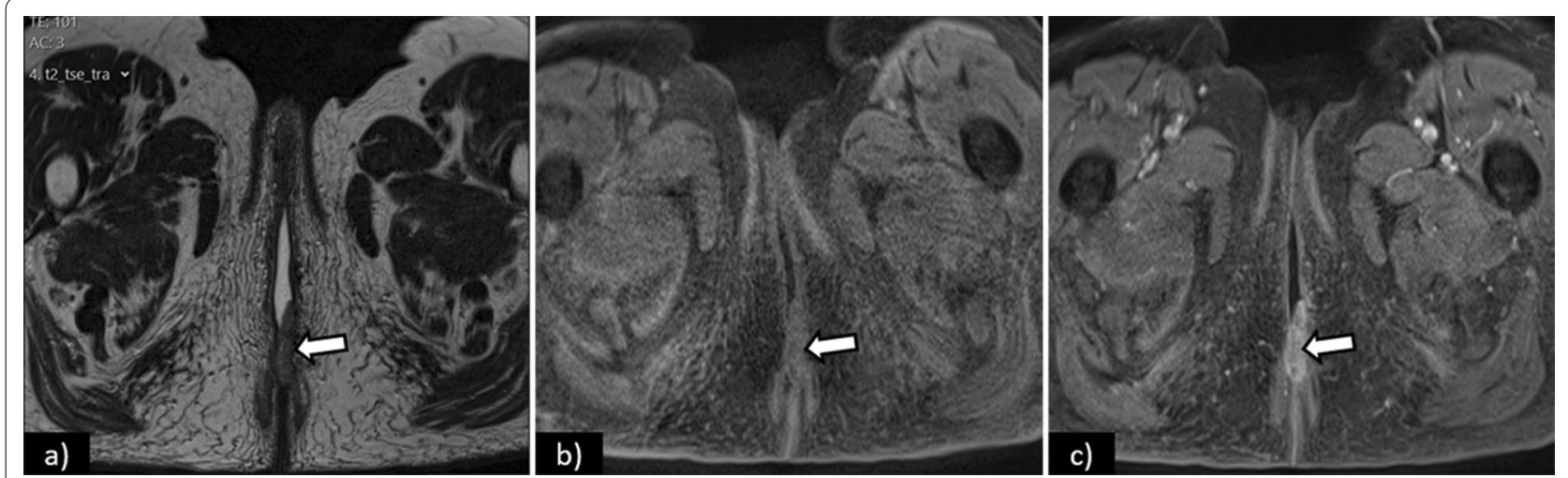

Fig. 3 Axial T2WI (a), axial T1WI fat saturation before (b), and after gadolinium (c) shows a vulvar tumour measuring $2.5 \mathrm{~cm}$, corresponding to FIGO stage IB. Note the increased conspicuity of the tumour in the contrast-enhanced sequence (c)

extracapsular spread (Fig. 6). This last criterion is a result of the significantly worse prognosis of node metastases with extracapsular spread, which are associated with a five-year overall survival of $34 \%$ versus $66 \%$ in patients with intranodal metastases [44].
- Stage IIIA1-1 lymph node metastasis ( $\geq 5 \mathrm{~mm})$.

- Stage IIIA2-1-2 lymph node metastasis(es) $(<5 \mathrm{~mm})$.

- Stage IIIB1-2 or more lymph nodes metastases ( $\geq 5 \mathrm{~mm})$. 


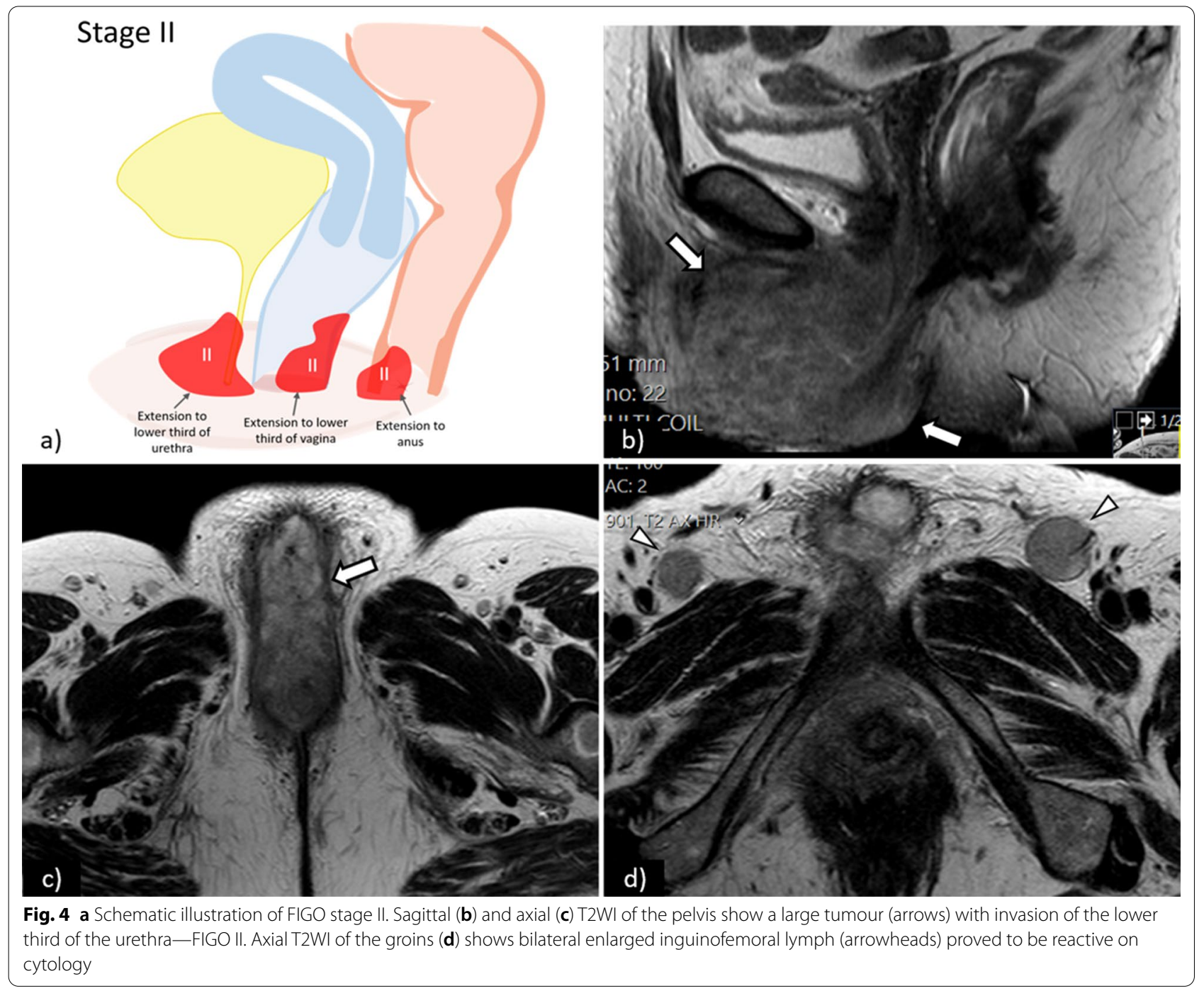

- Stage IIIB2-3 or more lymph nodes metastases $(<5 \mathrm{~mm})$.

- Stage IIIC-Positive nodes with extracapsular spread.

The risk of lymph node metastases is associated with primary tumour size, depth of stromal invasion, and the presence of lymphovascular space invasion. Vulvar carcinoma spreads via the lymphatic system primarily to the superficial inguinal nodes, as well as to the deep inguinal nodes (also known as deep femoral nodes), which are considered as regional sites. The subsequent involvement of pelvic lymph nodes is considered as distant metastasis. Lateral vulvar carcinomas drain to the ipsilateral inguinal lymph nodes, although lesions at or within $1 \mathrm{~cm}$ of the midline can drain to one or both sides [12, 27].

Pelvic lymph nodes are rarely involved in the absence of ipsilateral inguinal lymph node involvement, and an exception is made to some midline vulvar carcinomas and tumours with invasion of the vagina, bladder, or anus (above the dentate line) that may rarely spread directly to the pelvic lymph nodes (via the internal pudendal chain and internal iliac chain) [7].

Regional lymph node metastatic spread is the most important prognostic factor in vulvar cancer and determines the treatment choice $[34,46]$. As part of the latest revision in the FIGO staging system, not only the number of metastatic lymph nodes, but also metastasis size and the presence/absence of extra-nodal spread, should be stated by the pathologist.

The most well-accepted MRI criterion for regional lymph node metastasis is short axis $>1 \mathrm{~cm}$. Other features may be helpful, especially when combined, namely:irregular contour, round shape, presence of necrosis, loss of fatty hilum, and a ratio of 


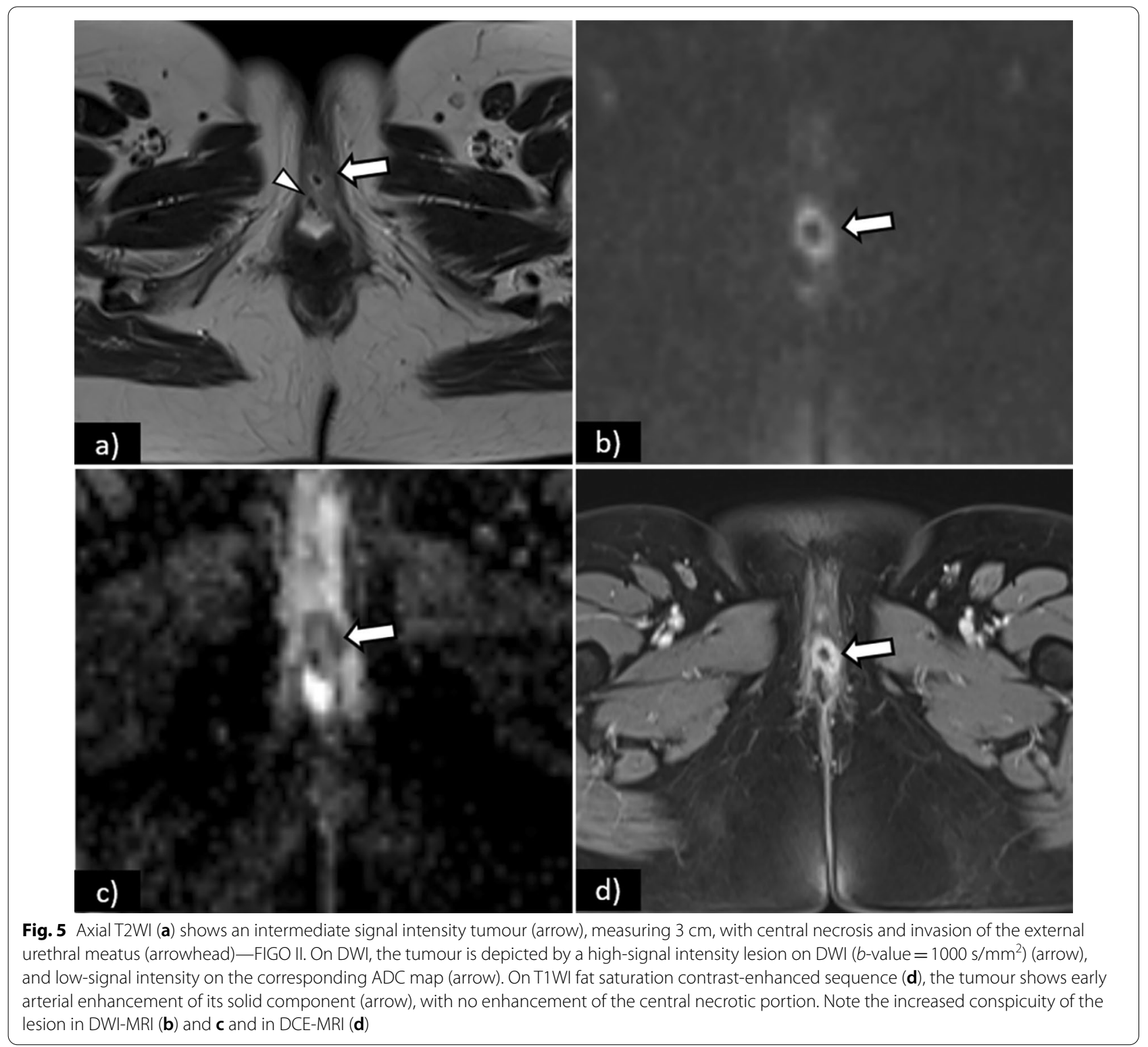

short-to-long-axis diameter $\geq 0.75$. Some of these features are shown in Figs. 7 and 8.

In case of discrepancy between positive radiological findings for inguinal lymph node metastasis (depicted at MRI, US, CT or PET/CT) and negative cytological/histological results following biopsy, the FIGO stage cannot be certainly estimated, and the multidisciplinary board must deliberate the most appropriate management for each case. If there is a strong radiological suspicion, a second ultrasound-guided lymph node biopsy may be performed using fusion virtual navigation systems that fuse real-time ultrasound images with previously acquired cross-sectional images using $\mathrm{CT}$, MRI, SPECT/CT or PET/CT [47-49].

\section{FIGO stage IV}

Stage IV comprises locally or regionally advanced disease (IVA) and distant disease (IVB).

- Stage IVA1-tumour invades upper $2 / 3$ of urethra and/or vagina, bladder mucosa, rectal mucosa or is fixed to pelvic bone (Fig. 9).

- Stage IVA2-fixed or ulcerated inguinofemoral lymph nodes (Fig. 10). 


\section{Stage III}
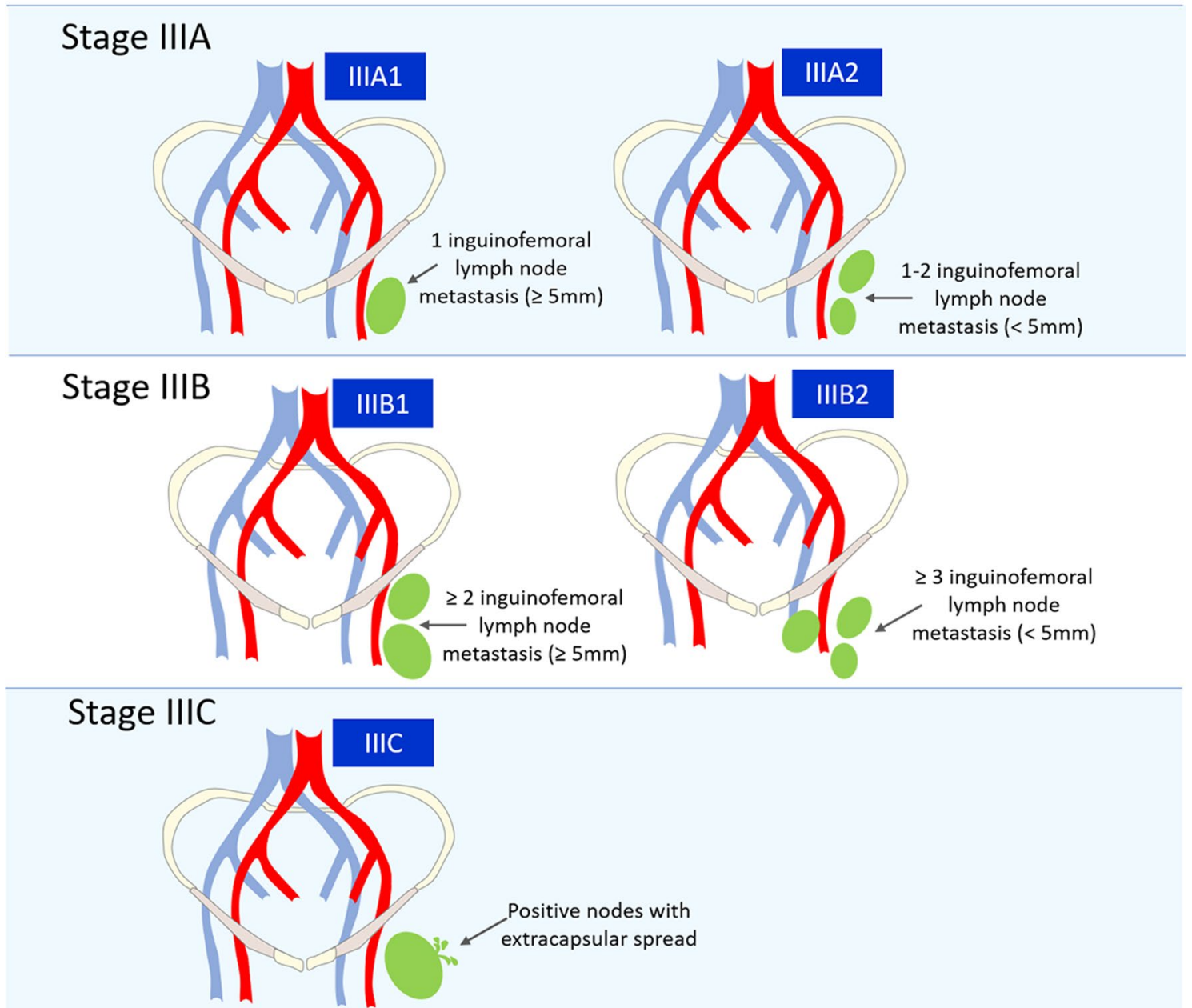

Fig. 6 Schematic illustration of FIGO stage III

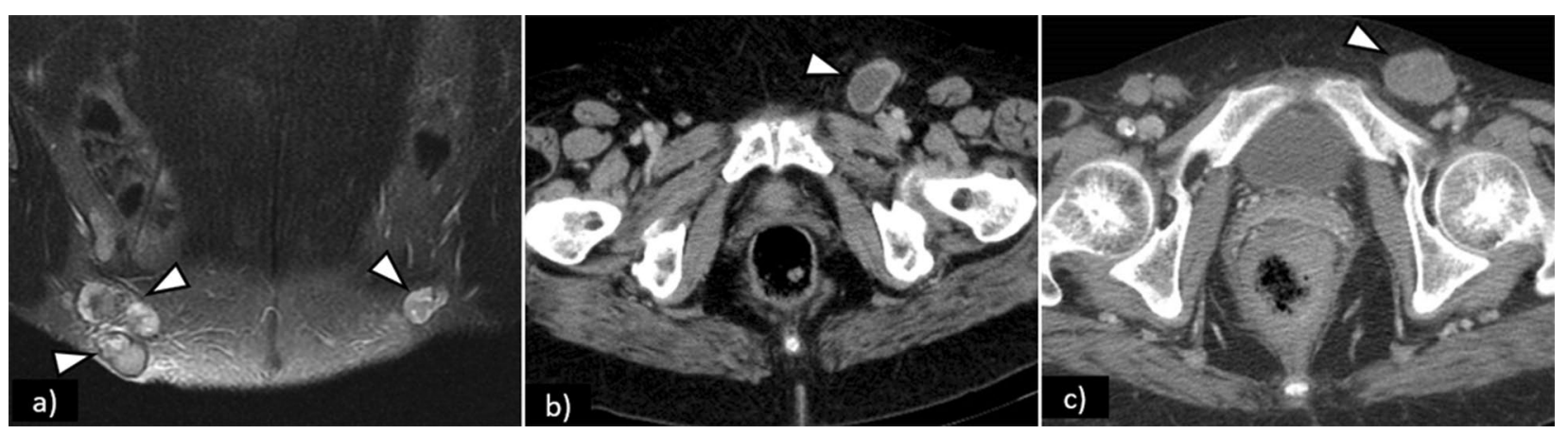

Fig. 7 Pathologically proven inguinofemoral lymph node metastasis in different patients (FIGO III): a coronal fat saturation T2WI shows bilateral enlarged heterogeneous lymph nodes with necrotic changes depicted by intra-nodal high-signal intensity areas; $\mathbf{b}$ axial CT shows left inguinofemoral enlarged node with low-attenuation necrotic centre; $\mathbf{c}$ axial CT shows heterogeneous enlarged left inguinofemoral node 

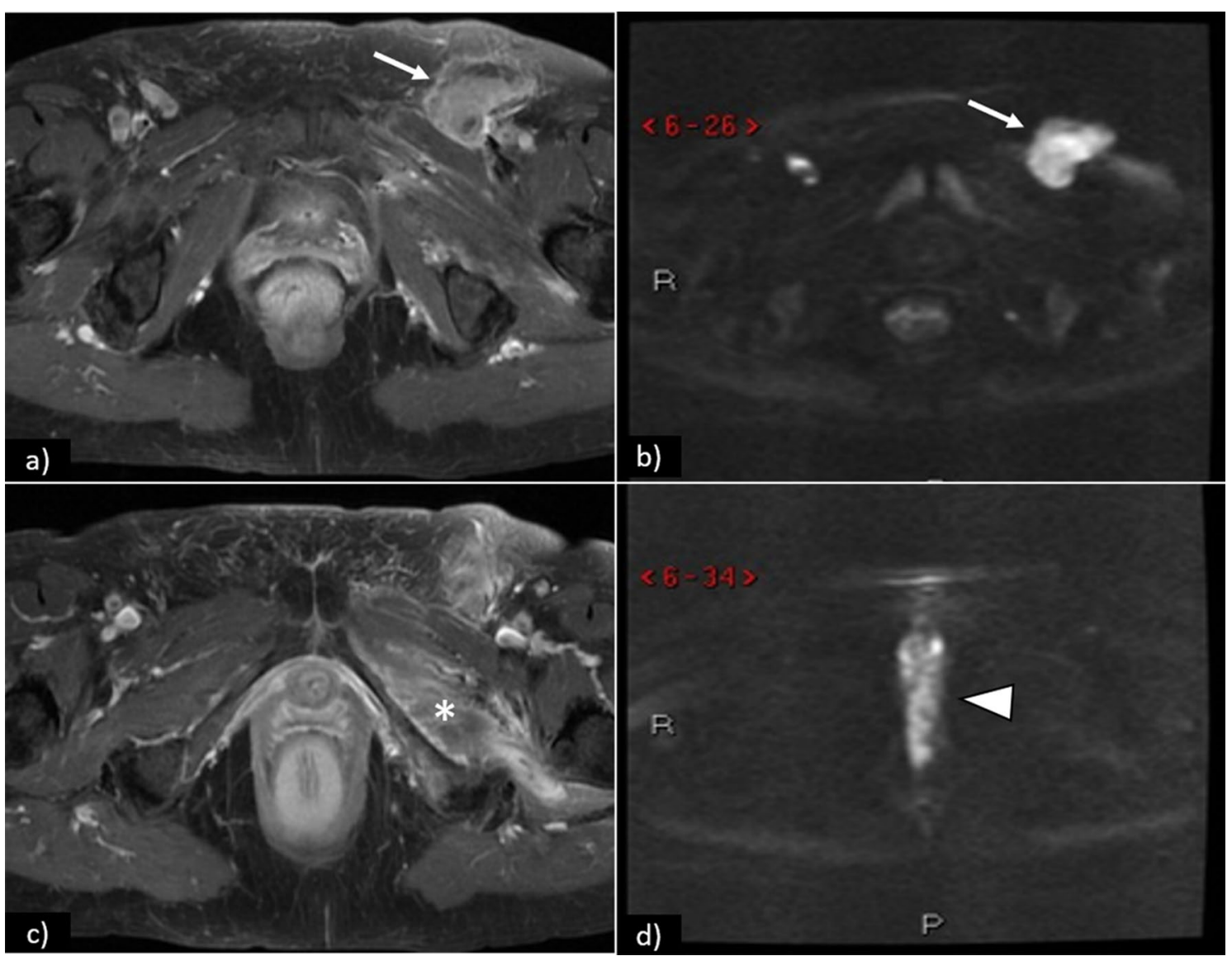

Fig. 8 Axial contrast-enhanced T1WI a shows left inguinal lymphadenopathy (arrow) with restricted diffusion (arrow) on DWI (b). At different levels, axial contrast-enhanced T1WI (c) depicts left external obturator muscle involvement $\left({ }^{*}\right)$, and DWI (d) shows restricted diffusion of the primary vulvar tumour (arrowhead)

- Stage IVB — any distant metastasis, including pelvic lymph nodes (Fig. 11).

Similar to FIGO stage II, invasion of regional pelvic structures can be depicted by an intermediate-signal intensity on T2WI disrupting the low-signal intensity of the upper two-thirds of the vagina or the upper twothirds of the urethra. Invasion of the bladder/rectal mucosal is diagnosed if an intermediate signal intensity tumour on T2WI disrupts low-signal intensity bladder or rectal wall and extends into the mucosa or the lumen [27]. DCE-MR can assist in better delineating tumour invasion of the nearby structures [12].

Distant metastasis is a rare occurrence in vulvar cancer, often preceded by one or more local recurrences. Most frequently involved sites include lung, liver, bone, lymph nodes (axillary, thoracic, and paraaortic), and skin $[46,50]$. In these cases, the prognosis is very poor with a two-year overall survival rate of $11.3 \%$ and a median survival from first diagnosis of metastases of only 5.6 months [50].

\section{Vulvar cancer: management and treatment}

Clinical and radiologic assessment of the inguinal regions (either by ultrasound, CT, PET/CT, or MRI) are needed to detect possible metastatic lymph node, which should then be analysed by FNA or core biopsy whenever this additional information impacts the primary treatment choice. Locally or regionally advanced-stage disease (with histologically proven regional lymph node metastasis) should be further staged with contrast-enhanced CT of the thorax, abdomen, and pelvis [5].

\section{Local treatment}

Local treatment of early stage vulvar carcinoma consists of radical local excision [5]. 


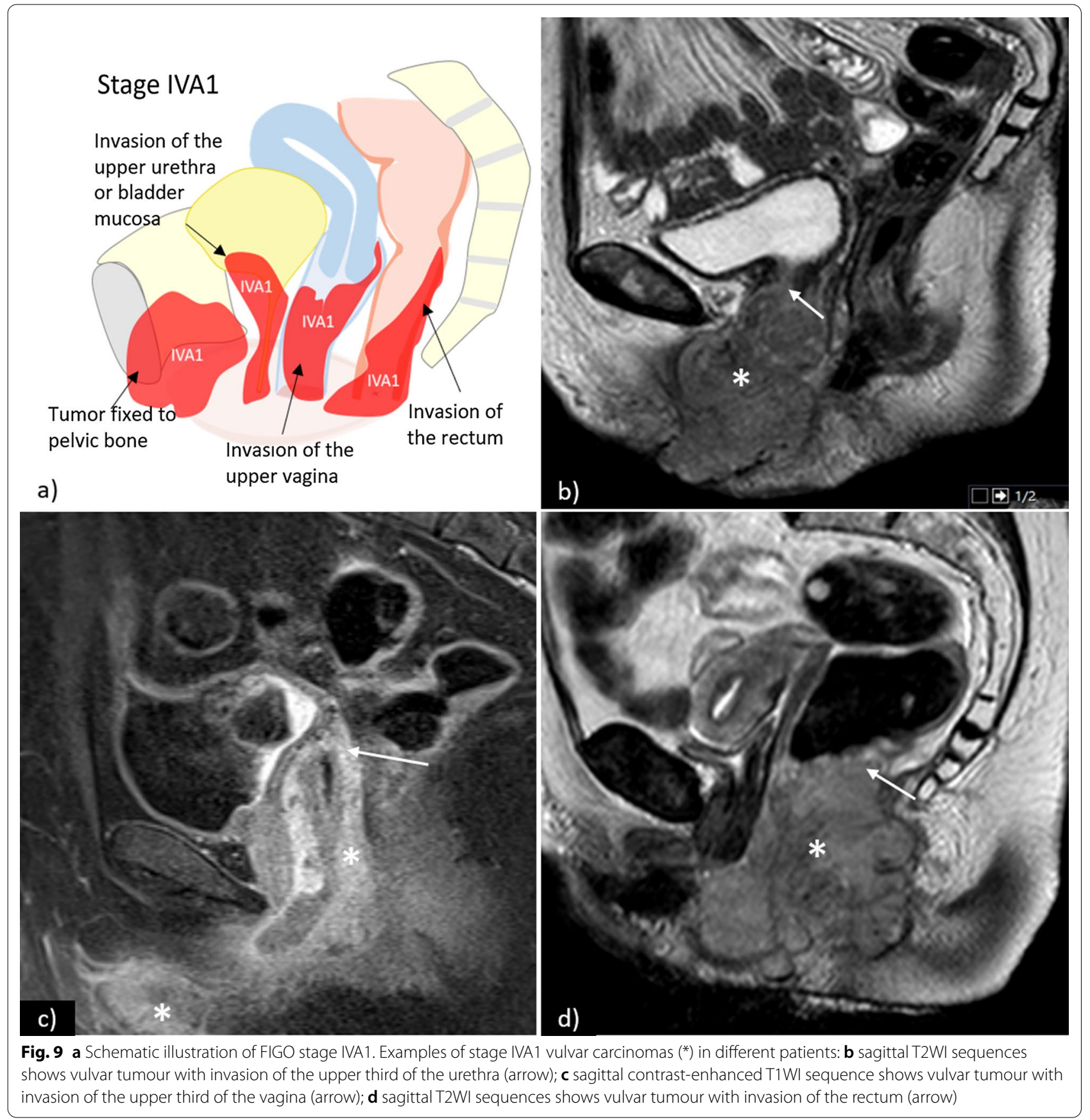

Surgical excision margins of at least $1 \mathrm{~cm}$ are advised. In cases of close proximity between the tumour and the clitoris, urethra, or anus, smaller margins may be considered in an attempt to preserve their function. If surgical margins are close $(<8 \mathrm{~mm})$ or positive, a second resection should be attempted. In cases of persistent positive margins or if the patient is not eligible for a second surgical intervention, adjuvant local RT is advised [5, 33, 51].
Treatment of advanced-stage vulvar cancer involves multiple treatment modalities including surgery, radiotherapy (RT), and chemotherapy. The optimal choice should be discussed in a multidisciplinary setting [ 5 , 33].

\section{Groin treatment}

IFLD is not required for stage IA disease due to its low risk of lymph node metastasis [5, 52-55]. 


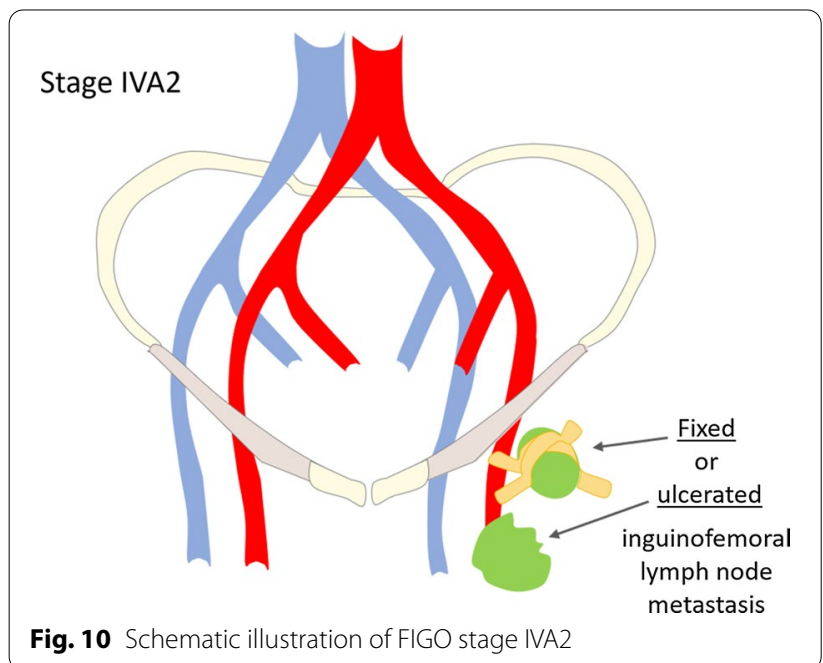

For tumours greater than stage IA (i.e. with stromal invasion $>1 \mathrm{~mm}$ ) groin treatment should be performed. Depending on tumour size, SLN procedure (for tumours $<4 \mathrm{~cm}$ ) or IFLD (for tumours $\geq 4 \mathrm{~cm}$ or in case of multifocal disease) is recommended. In cases of a positive SLN with a node metastasis $<2 \mathrm{~mm}$, RT has shown to be a safe alternative to ILFD [56].

Contralateral IFLD may be performed when there is ipsilateral node involvement [5]. Postoperative RT to the groin is advocated for cases with more than 1 metastatic lymph node and/or in the presence of extracapsular lymph node spread [5].

\section{Unresectable disease}

In advanced-stage unresectable disease (larger stage II and stage IVA tumours), definitive chemoradiation is the treatment of choice. In selected cases, neoadjuvant chemoradiation should be considered [5].

\section{Recurrent disease}

Recurrences of vulvar carcinoma are common and usually occur within the first 2 years after initial presentation [12]. Vulvar and perineal region are the most frequent sites of local recurrences. Life-long follow-up after primary surgical treatment is advised and includes clinical examination of the vulva and groins (despite the low sensitivity of palpation in identifying groin metastasis, since available data does not support the routine use of imaging of the groins in follow-up) [5]. Clinical suspicion should be followed by biopsy and imaging work-up [57].

Local recurrences should be treated as primary tumours with wide local excision and inguinofemoral lymphadenectomy (if not previously performed), with or without postoperative radiotherapy [5]. CT of the chest and abdomen or PET/CT is recommended to assess the

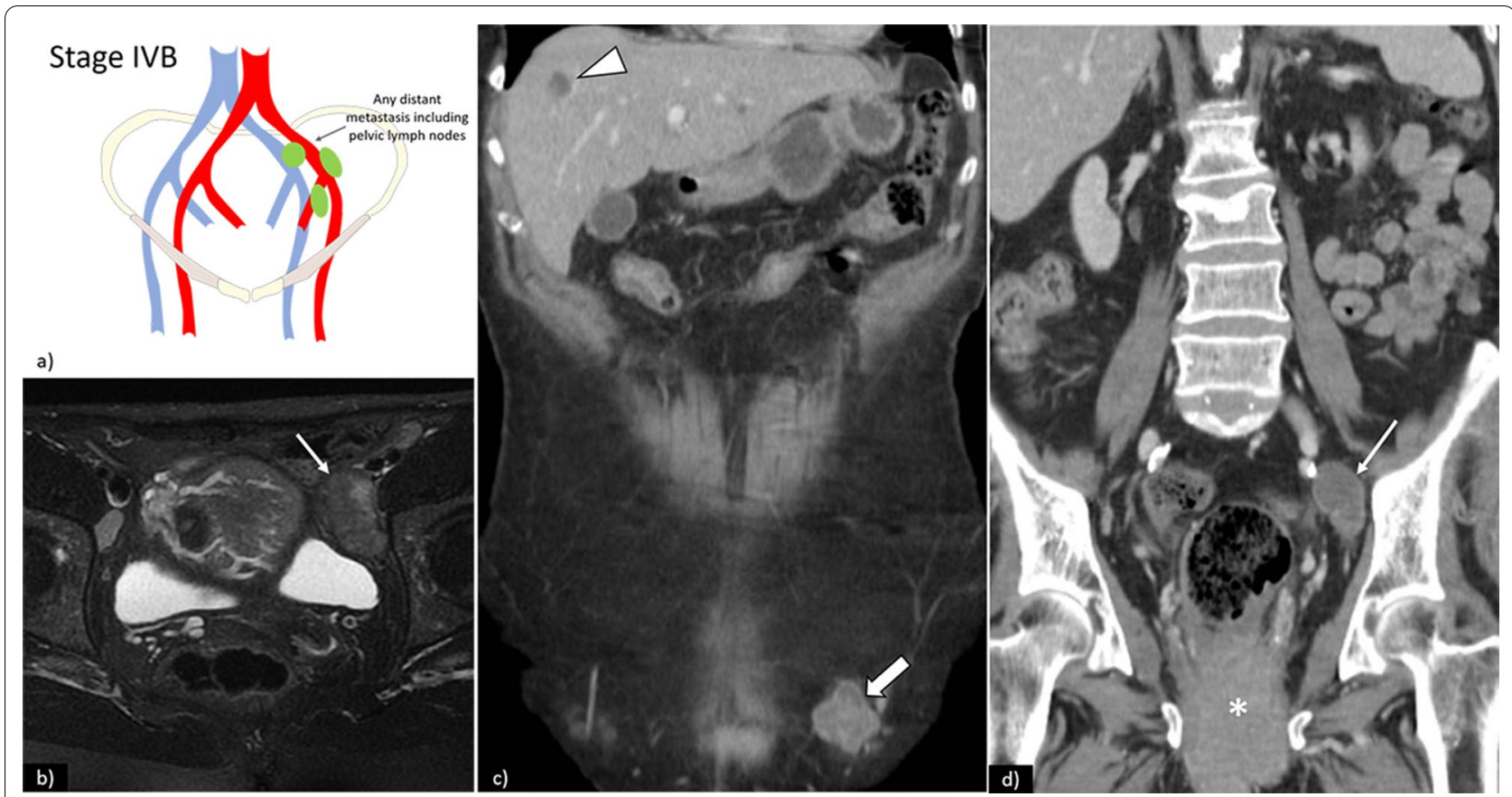

Fig. 11 a Schematic illustration of FIGO stage IVB. Axial fat saturation T2WI shows left external iliac lymph node metastasis (arrow), depicted by increased lymph node size and heterogeneity. In another patient, coronal CT (c) and (d) shows vulvar tumour $\left(^{*}\right)$ with inguinal lymph node involvement (open arrow), internal iliac lymph node involvement (arrow), and hepatic metastasis (arrowhead) 
presence of additional metastases [5]. MRI is useful to examine the extent of the local recurrence and to plan further treatment.

In groin recurrence, restaging by $\mathrm{CT}$ (or PET/CT) of the chest, abdomen and pelvis is advocated and the preferred treatment is radical excision (when possible), followed by adjuvant radiation in radiotherapy-naive patients. When surgery is not possible, definitive chemoradiation is recommended [5].

If distant metastases are present, systemic (palliative) therapy should be considered along with local radiotherapy for control of locoregional disease [33].

\section{Summary}

The authors' recommendations on the initial staging of vulvar cancer are in accordance with the latest revision of the FIGO classification (2009). These ESUR guidelines were developed by the Female Pelvis Imaging Working Group, with the main purpose of standardising MRI protocols, interpretation, and reporting, ultimately aiming to reduce ambiguity and improve the contribution of radiology in the staging and management of these patients.

\begin{abstract}
Abbreviations
3DT1WI FS: Three-dimensional spoiled gradient-echo fat-suppressed T1-weighted imaging; ADC: Apparent diffusion coefficient; AJCC: American Joint Commission on Cancer; DCE: Dynamic contrast-enhanced; DWI: Diffusion-weighted imaging; ESGO: European Society of Gynaecological Oncology; ESUR: European Society of Urogenital Radiology; FDG-PET: 18F fluorodeoxyglucose positron emission tomography; FIGO: International Federation of Gynaecology and Obstetrics; FNA: Fine-needle aspiration; FOV: Field-of-view; HPV: Human papillomavirus; IFLD: Inguinofemoral lymph node dissection; rFOV: Reduced field-of-view; RT: Radiotherapy; SCC: Squamous cell carcinoma; SLN: Sentinel lymph node; SPECT/CT: Single photon emission computed tomography/computed tomography; T1WI:T1-weighted imaging; T2Wl: T2-weighted imaging; TNM: Primary tumour ( $T$ ), regional lymph nodes (N), distant metastases (M); UICC: Union of International Cancer Control; VIN: Vulvar intraepithelial neoplasia; WHO: World Health Organization.
\end{abstract}

\section{Acknowledgements}

The committee would like to thank the following ESUR members for participating in the survey: Teresa Margarida Cunha, Rita Lucas, Cristina Maciel, Portugal; Olivera Nikolic, Marijana Basta Nikolić, Serbia; Henrik Leonhardt, Sweden; M. Milagros Otero-Garcia, Laura Buñesch Villalba, Spain; Charis Bourgioti, Athina C. Tsili, Greece; Stephanie Nougaret, Laure Fournier, France; Lucia Manganaro, Benedetta Gui, Italy; Celine Alt, Germany; Rosemarie Forstner, Austria; Evis Sala, Nishat Bharwani, Carolina Lopez, United Kingdom; Yulia Lakhman, USA; Aki Kido, Japan. The authors of this manuscript declare no relationships with any companies whose products or services may be related to the subject matter of the article. The authors state that this work has not received any funding. No complex statistical methods were necessary for this paper. Institutional Review Board approval was not required because this manuscript presents guidelines only and has not required the use of patient-sensitive data. Some study subjects or cohorts have not been previously reported. Methodology: Guidelines based on expert opinion and literature review.

\section{Authors' contributions}

All eight authors have read and approved the manuscript. ON involved in questionnaire's design, partial writing of the manuscript, and collection of cases. FAS involved in partial writing of the manuscript, language and grammar editing, collection of cases and preparation of figures, preparation of tables, review concept, and design. TMC involved in partial writing of the manuscript, collection of cases, and supervision of work. MBN involved in partial writing of the manuscript. MMOG involved in partial writing of the manuscript. BG involved in partial writing of the manuscript. SN involved in partial writing of the manuscript, collection of cases, and preparation of figures. $\mathrm{HL}$ involved questionnaire's design, in partial writing of the manuscript and supervision of the work. All authors read and approved the final manuscript.

\section{Funding}

Granted by the Insights into Imaging Editorial Office.

Availability of data and materials

Not applicable.

\section{Declarations}

Ethics approval and consent to participate

Not applicable.

\section{Consent for publication}

Not applicable.

\section{Competing interests}

The authors declare that they have no competing interests.

\section{Author details}

${ }^{1}$ Center of Radiology, Clinical Center of Vojvodina, Faculty of Medicine, University of Novi Sad, Hajduk Veljkova 1-9, 21000 Novi Sad, Serbia. ${ }^{2}$ Department of Radiology, Centro Hospitalar Universitário de Lisboa Central, Alameda Santo António Dos Capuchos, $1169-050$ Lisboa, Portugal. ${ }^{3}$ Department of Radiology, Instituto Português de Oncologia de Lisboa Francisco Gentil, R. Prof. Lima Basto, 1099-023 Lisbon, Portugal. ${ }^{4}$ Hospital Universitario de Vigo (CHUVI), Estrada Clara Campoamor 341, 36312 Vigo, Spain. ${ }^{5}$ Fondazione Policlinico Universitario A. Gemelli IRCCS, UOC Radiologia Generale Ed Interventistica Generale, Area Diagnostica Per Immagini, Dipartimento Diagnostica Per Immagini, Radioterapia Oncologica ed Ematologia, Rome, Italy. ${ }^{6}$ Department of Radiology, Montpellier Cancer Institute, 15 INSERM, Montpellier Cancer Research Institute, U1 194, University of Montpellier, 208 Avenue des Apothicaires, 34295 Montpellier, France. ${ }^{7}$ Department of Radiology, Institute of Clinical Sciences, Sahlgrenska University Hospital, Bruna straket 11B, 413 45 Gothenburg, Sweden.

Received: 14 March 2021 Accepted: 11 August 2021

Published online: 22 September 2021

References

1. Rogers LJ, Cuello MA (2018) Cancer of the vulva. Int J Gynecol Obstet 143:4-13

2. Miccò M, Sala E, Lakhman Y, Hricak H, Vargas HA (2015) Imaging features of uncommon gynecologic cancers. AJR Am J Roentgenol 205:1346-1359

3. Tan J, Chetty N, Kondalsamy-Chennakesavan S et al (2012) Validation of the FIGO 2009 staging system for carcinoma of the vulva. Int J Gynecol Cancer 22:498-502

4. Sobin LH, Gospodarowicz MK, Wittekind C (2010) TNM classification of malignant tumors, 7th edn. In: International union against cancer. New York

5. Oonk MHM, Planchamp F, Baldwin P et al (2017) European society of gynaecological oncology guidelines for the management of patients with Vulvar cancer. Int J Gynecol Cancer 27:832-837

6. Chan JK, Sugiyama V, Pham H et al (2007) Margin distance and other clinico-pathologic prognostic factors in vulvar carcinoma: a multivariate analysis. Gynecol Oncol 104:636-641

7. Sohaib SAA, Moskovic EC (2003) Imaging in vulval cancer. Best Pract Res Clin Obstet Gynaecol 17:543-556

8. Vulvar Cancer (Squamous Cell Carcinoma) Version 2 (2019) In NCCN Clinical Practice Guidelines in Oncology. https://www.nccn.org/professionals/ physician_gls/pdf/vulvar_blocks.pdf. Accessed 08 Feb 2021 
9. Lakhman Y, Vargas HA, Reinhold C et al. (2020) American College of Radiology ACR Appropriateness Criteria. https://acsearch.acr.org/docs/31024 02/Narrative/. Acessed 08 Feb 2021.

10. Sohaib AAA, Richards PS, Ind T et al (2010) MR imaging of carcinoma of the vulva. AJR Am J Roentgenol 178:373-377

11. Kataoka MY, Sala E, Baldwin P et al (2010) The accuracy of magnetic resonance imaging in staging of vulvar cancer: a retrospective multi-centre study. Gynecol Oncol 117:82-87

12. Viswanathan C, Kirschner K, Truong M, Balachandran A, Devine C, Bhosale P (2013) Multimodality imaging of vulvar cancer: staging, therapeutic response, and complications. AJR Am J Roentgenol 200:1387-1400

13. Moskovic EC, Shepherd JH, Barton DPJ, Trott PA, Nasiri N, Thomas JM (1999) The role of high resolution ultrasound with guided cytology of groin lymph nodes in the management of squamous cell carcinoma of the vulva: a pilot study. Br J Obstet Gynaecol 106:863-867

14. Hall TB, Barton DPJ, Trott PA et al (2003) The role of ultrasound-guided cytology of groin lymph nodes in the management of squamous cell carcinoma of the vulva: 5 -year experience in 44 patients. Clin Radiol 58:367-371

15. Hawnaur JM, Reynolds K, Wilson G, Hillier V, Kitchener HC (2002) Identification of inguinal lymph node metastases from vulval carcinoma by magnetic resonance imaging: an initial report. Clin Radiol 57:995-1000

16. Bipat S, Fransen GA, Spijkerboer AM et al (2006) Is there a role for magnetic resonance imaging in the evaluation of inguinal lymph node metastases in patients with vulva carcinoma? Gynecol Oncol 103:1001-1006

17. Singh K, Orakwue CO, Honest H, Balogun M, Lopez C, Luesley DM (2006) Accuracy of magnetic resonance imaging of inguinofemoral lymph nodes in vulval cancer. Int J Gynecol Cancer 16:1179-1183

18. Cohn DE, Dehdashti F, Gibb RK et al (2002) Prospective evaluation of positron emission tomography for the detection of groin node metastases from vulvar cancer. Gynecol Oncol 85:179-184

19. De Hullu JA, Pruim J, Qué TH et al (1999) Noninvasive detection of inguinofemoral lymph node metastases in squamous cell cancer of the vulva by L-[1-11C]-tyrosine positron emission tomography. Int J Gynecol Cancer 9:141-146

20. Crivellaro C, Guglielmo P, De Ponti E et al (2017) 18F-FDG PET/CT in preoperative staging of vulvar cancer patients: Is it really effective? Medicine (Baltimore) 96:e7943

21. Andersen K, Zobbe V, Thranov IR, Pedersen KD (2015) Relevance of computerized tomography in the preoperative evaluation of patients with vulvar cancer: a prospective study. Cancer Imaging 15:8

22. Pounds R, O'Neill D, Subba K et al (2020) The role of preoperative computerized tomography (CT) scan of the pelvis and groin in the management of clinically early staged vulva squamous cell carcinoma. Gynecol Oncol 157:444-449

23. Bohlin KS, Bruno A-K, von Knorring C, Rahm C, Leonhardt H (2021) Accuracy of computerized tomography in the preoperative evaluation of metastases in primary vulvar cancer: a population-based study. Gynecol Oncol 161:449-453

24. Selman TJ, Luesley DM, Acheson N, Khan KS, Mann CH (2005) A systematic review of the accuracy of diagnostic tests for inguinal lymph node status in vulvar cancer. Gynecol Oncol 99:206-214

25. Land R, Herod J, Moskovic E et al (2006) Routine computerized tomography scanning, groin ultrasound with or without fine needle aspiration cytology in the surgical management of primary squamous cell carcinoma of the vulva. Int J Gynecol Cancer 16:312-317

26. Nooij LS, Ongkiehong PJ, Van Zwet EW et al (2015) Groin surgery and risk of recurrence in lymph node positive patients with vulvar squamous cell carcinoma. Gynecol Oncol 139:458-464

27. Burger MPM, Hollema H, Emanuels AG, Krans M, Pras E, Bouma J (1995) The importance of the groin node status for the survival of T1 and T2 vulval carcinoma patients. Gynecol Oncol 57:327-334

28. Te Grootenhuis NC, Van Der Zee AGJ, Van Doorn HC et al (2016) Sentinel nodes in vulvar cancer: long-term follow-up of the GROningen INternational Study on Sentinel nodes in Vulvar cancer (GROINSS-V) i. Gynecol Oncol 140:8-14

29. Covens A, Vella ET, Kennedy EB, Reade CJ, Jimenez W, Le T (2015) Sentinel lymph node biopsy in vulvar cancer: systematic review, meta-analysis and guideline recommendations. Gynecol Oncol 137:351-361
30. Collarino A, Fuoco V, Garganese G et al (2020) Lymphoscintigraphy and sentinel lymph node biopsy in vulvar carcinoma: update from a European expert panel. Eur J Nucl Med Mol Imaging 47:1261-1274

31. Maglinte DDT, Hale DS, Sandrasegaran K (2013) Comparison between dynamic cystocolpoproctography and dynamic pelvic floor MRI: pros and cons: which is the "functional" examination for anorectal and pelvic floor dysfunction Abdom. Imaging 38:952-973

32. Serrado MA, Horta M, Cunha TM (2019) State of the art in vulvar cancer imaging. Radiol Bras 52:316-324

33. Boone JM, Mahesh M, Gingold EL, Seibert JA (2016) A call for the structured physicist report. J Am Coll Radiol 13:307-309

34. Flusberg M, Ganeles J, Ekinci T et al (2017) Impact of a structured report template on the quality of $\mathrm{CT}$ and MRI reports for hepatocellular carcinoma diagnosis. J Am Coll Radiol 4:1206-1211

35. Gassenmaier S, Armbruster M, Haasters F et al (2017) Structured reporting of MRI of the shoulder: improvement of report quality? Eur Radiol 27:4110-4119

36. Koh WJ, Greer BE, Abu-Rustum NR et al (2017) Vulvar cancer, version 1.2017: Clinical practice guidelines in oncology. J Natl Compr Cancer Netw 15:92-120

37. Kim KW, Shinagare AB, Krajewski KM et al (2013) Update on imaging of vulvar squamous cell carcinoma. AJR Am J Roentgenol 201:147-157

38. SEER Cancer Statistics Factsheets: Vulvar Cancer (2021) https://seer. cancer.gov/statfacts/html/vulva.html. Accessed 09 Jan 2021

39. Oldan J, Sullivan S (2018) Positron emission tomography-computed tomography for inguinal nodes in vulvar cancer. World J Nucl Med 17:139-144

40. Kamran MW, O'Toole F, Meghen K, Wahab AN, Saadeh FA, Gleeson N (2014) Whole-body [18F]fluoro-2-deoxyglucose positron emission tomography scan as combined PET-CT staging prior to planned radical vulvectomy and inguinofemoral lymphadenectomy for squamous vulvar cancer: a correlation with groin node metastasis. Eur J Gynaecol Oncol 35:230-235

41. Solon JG, Power C, Al-Azawi D, Duke D, Hill ADK (2012) Ultrasoundguided core biopsy: an effective method of detecting axillary nodal metastases. J Am Coll Surg 214:12-17

42. Houssami N, Ciatto S, Turner RM, Cody HS, MacAskill P (2011) Preoperative ultrasound-guided needle biopsy of axillary nodes in invasive breast cancer: meta-analysis of its accuracy and utility in staging the axilla. Ann Surg 254:243-25

43. Ciatto S, Brancato B, Risso G et al (2007) Accuracy of fine needle aspiration cytology (FNAC) of axillary lymph nodes as a triage test in breast cancer staging. Breast Cancer Res Treat 103:85-91

44. Van Rijk MC, Deurloo EE, Nieweg OE et al (2006) Ultrasonography and fine-needle aspiration cytology can spare breast cancer patients unnecessary sentinel lymph node biopsy. Ann Surg Oncol 13:31-35

45. Britton PD, Goud A, Godward S et al (2009) Use of ultrasound-guided axillary node core biopsy in staging of early breast cancer. Eur Radiol 19:561-569

46. Abe H, Schmidt RA, Kulkarni K, Sennett CA, Mueller JS, Newstead GM (2009) Axillary lymph nodes suspicious for breast cancer metastasis: Sampling with US-guided 14-gauge core-needle biopsy: clinical experience in 100 patients. Radiology 250:41-49

47. Pecorelli S (2009) International journal of gynecology and obstetrics revised FIGO staging for carcinoma of the vulva, cervix, and endometrium. Int J Gynecol Obs 105:103-104

48. Tabbaa ZM, Gonzalez J, Sznurkowski JJ, Weaver AL, Mariani A, Cliby WA (2012) Impact of the new FIGO 2009 staging classification for vulvar cancer on prognosis and stage distribution. Gynecol Oncol 127:147-152

49. Van Der Steen S, Van De Nieuwenhof HPV, Massuger L, Bulten J, De Hullu JA (2010) New FIGO staging system of vulvar cancer indeed provides a better reflection of prognosis. Gynecol Oncol 119:520-525

50. Slomovitz BM, Coleman RL, Oonk MHM, Van Der Zee A, Levenback C (2015) Update on sentinel lymph node biopsy for early-stage vulvar cancer. Gynecol Oncol 138:472-477

51. Maggino T, Landoni F, Sartori E et al (2000) Patterns of recurrence in patients with squamous cell carcinoma of the vulva: a multicenter CTF study. Cancer 89:116-122 
52. Garganese G, Bove S, Zagaria L et al (2019) Fusion of ultrasound and 3D single-photon-emission computed tomography/computed tomography to identify sentinel lymph nodes in vulvar cancer: feasibility study. Ultrasound Obstet Gynecol 54:545-551

53. Garganese G, Bove S, Fragomeni S et al (2021) Real-time ultrasound virtual navigation in 3D PET/CT volumes for superficial lymph node evaluation: an innovative fusion examination. Ultrasound Obstet Gynecol. https://doi.org/10.1002/uog.23613

54. Gentileschi S, Albanese R, Pino V et al (2019) SPECT/CT and fusion ultrasound to target the efferent groin lymph node for lymphatic surgery. Microsurgery 39:605-612
55. Prieske K, Haeringer N, Grimm D et al (2016) Patterns of distant metastases in vulvar cancer. Gynecol Oncol 142:427-434

56. Mitra S, Kumar Sharma M, Kaur I et al (2018) Vulvar carcinoma: dilemma, debates, and decisions. Cancer Manag Res 10:61-68

57. Stehman FB, Look KY (2006) Carcinoma of the vulva. Obstet Gynecol 107:719-733

\section{Publisher's Note}

Springer Nature remains neutral with regard to jurisdictional claims in published maps and institutional affiliations.

\section{Submit your manuscript to a SpringerOpen ${ }^{\circ}$ journal and benefit from:}

- Convenient online submission

- Rigorous peer review

- Open access: articles freely available online

- High visibility within the field

- Retaining the copyright to your article

Submit your next manuscript at springeropen.com 